OPEN ACCESS

Edited by:

Yinghui Zhong,

Drexel University, United States

Reviewed by:

Lija Swain,

Tufts Medical Center, United States

Atsushi Tanaka,

Saga University, Japan

*Correspondence:

Yong $\mathrm{Li}$

liyong606@126.com

Specialty section:

This article was submitted to

Cardiovascular Therapeutics,

a section of the journal

Frontiers in Cardiovascular Medicine

Received: 28 May 2021

Accepted: 23 August 2021

Published: 23 September 2021

Citation:

Bao L, Gao X, Xie K and Li Y (2021) Is

There a Diabetes-Kidney-Heart

Continuum? Perspectives From the

Results of the Cardiovascular and Renal Outcome Clinical Trials With

SGLT2 Inhibitors.

Front. Cardiovasc. Med. 8:716083

doi: 10.3389/fcvm.2021.716083

\section{Is There a Diabetes-Kidney-Heart Continuum? Perspectives From the Results of the Cardiovascular and Renal Outcome Clinical Trials With SGLT2 Inhibitors}

\author{
Liwen Bao, Xiufang Gao, Kun Xie and Yong Li * \\ Department of Cardiology, Fuden University Affiliated Huashan Hospital, Shanghai, China
}

Heart failure is associated with a substantial risk of mortality and morbidity. Findings from recent cardiovascular outcome trials have shown promise for sodium-glucose cotransporter-2 (SGLT2) inhibitors in preventing heart failure in patients with type 2 diabetes mellitus (T2DM). Notably, the benefits of SGLT2 inhibitors were consistent despite the presence of risk factors like atherosclerosis. Increasing evidence suggests that SGLT2 inhibitors may confer their cardioprotective effects through multiple mechanisms, ranging from improving cardiac and vascular performance to metabolism. The reduction of heart failure risk by SGLT2 inhibitors may also be attributed to the preservation of renal function. Indeed, renal insufficiency is a frequent comorbidity of patients with heart failure and T2DM; hence, the natriuretic and kidney protective effects offered by SGLT2 inhibitors may contribute to limiting adverse cardiac outcomes. In this article, we discuss the latest findings from the cardiovascular and renal outcome trials, paying special attention to the interlink between heart and kidney function, and how effective treatment of heart failure-irrespective of T2DM diagnosis - may require agents that offer both cardiac and renal protection.

Keywords: heart failure, type 2 diabetes mellitus, renal insufficiency, CVOT, SGLT2 inhibitors

\section{INTRODUCTION}

Heart failure (HF) is a fatal disease that imparts a heavy burden on patients and healthcare systems. Approximately 26 million people are affected by HF worldwide, and its prevalence is rising each year (1). Atherosclerotic cardiovascular disease (ASCVD) is considered the leading cause of HF (2). While lipid-lowering and anti-platelet aggregation therapy have reduced the risks of major adverse cardiovascular events, such progress in coronary heart disease treatment has not brought about an improvement in the morbidity and mortality risks faced by patients with HF. Data from the US National Health Interview Survey, a study that examined 677,051 adults over a period of 11.8 years, showed a significant decrease in mortality rates in major cardiovascular disease (CVD), ischemic heart disease, and stroke, but not for patients with HF and arrhythmia (3).

Diminishing renal function is one of the most potent negative catalysts for HF. A decrease in estimated glomerular filtration rate (GFR) and albuminuria may determine the risk of progression to end-stage renal disease, and identify patients with type 2 diabetes mellitus (T2DM) at markedly higher risk of HF (4). Results from recent cardiovascular outcome trials have suggested that the 
benefits of sodium-glucose cotransporter 2 (SGLT) inhibitors (e.g., dapagliflozin, empagliflozin and canagliflozin) derived by patients with HF can be attributed to an improvement in renal function (5-8). A systematic review and meta-analysis of these trials also uncovered that patients without ASCVD but with multiple risk factors like T2DM experienced similar cardiorenal benefits to those with ASCVD (9). Collectively, these data suggest that, besides ASCVD, other factors might be associated with the progression of HF.

We propose that the outcomes from the recent clinical trials with SGLT2 inhibitors have uncovered a link between diabetes, kidney, and heart function. This continuum may partially explain the cardiovascular and renal benefits associated with SGLT2 inhibitors in recent cardiovascular outcomes trials. Here, we provide an overview of the link between T2DM, HF and renal insufficiency, and discuss these associations in the context of cardiovascular and renal outcomes with SGLT2 inhibitors in patients with and without T2DM. We also explore the potential mechanisms underlying cardiorenal protection by SGLT2 inhibitors.

\section{T2DM AND HF}

T2DM is a well-known cause of HF independent of ASCVD (10). The underlying mechanisms include advanced glycation end-product accumulation, microvascular dysfunction, inflammation, and lipotoxicity (11). In HF cohorts, patients with T2DM experience poorer clinical outcomes than patients without T2DM (12-14). More importantly, metabolic disorders such as T2DM, rather than ASCVD, are largely responsible for the development of $\mathrm{HF}$ with preserved ejection fraction (HFpEF), a condition with a substantial unmet need for effective treatments (15).

\section{T2DM AND RENAL INSUFFICIENCY}

T2DM is considered one of the causes of renal impairment and, consequently, $\operatorname{HF}(16,17)$. During renal injury, persistent exposure to high glucose concentrations during T2DM results in increased expression and activity of SGLT2 in the proximal tubules, which in turn leads to a maladaptive increase in glucose and sodium reabsorption and decreased sodium delivery to the macula densa (18). In spite of the increased GFR, reduced exposure of the macula densa to sodium causes an afferent renal vasodilatory response and activation of the renin-angiotensinaldosterone system (RAAS) $(18,19)$. Due to long-term persistent water-sodium retention, activation of these pathways contributes to the development of renal dysfunction $(18,19)$. Indeed, studies have shown that dysglycemia is a risk factor for the development of renal complications in patients with T2DM, as approximately $33 \%$ of patients with T2DM develop chronic kidney disease (CKD) (20). T2DM-associated CKD has now become one of the leading causes of renal failure (21).

\section{RENAL INSUFFICIENCY AND HF}

Renal dysfunction itself increases the risk of HF. It has been suggested that a decline in estimated GFR is associated with higher risk for all-cause mortality and CVD (22). Cardiac and renal diseases may interact in a complex, bidirectional and interdependent manner through three central mechanisms: hemodynamic, neurohormonal, and CVD-associated mechanisms (23). Hemodynamic mechanisms include salt and water retention, which leads to fluid overload and eventually cardiac and renal venous congestion $(23,24)$. Neurohormonal mechanisms are mainly composed of the activation of both RAAS and the sympathetic nervous system (23). CVD-associated mechanisms comprise systemic and local inflammatory conditions that are due to the deregulated innate and adaptive immune system, bone-mineral and acid-base disorders, anemia, and cachexia (23).

\section{DISCUSSION}

\section{SGLT2 Inhibitors and Link Between T2DM, Heart and Kidney Function}

There is a complex and mutually reinforcing relationship between T2DM, the kidney, and heart. These interactions appear to confer intractable changes in volume regulation, sodium homeostasis, inflammation, and metabolism that typically lead to rapid decline and early mortality. Until the emergence of SGLT2 inhibitors, breaking this vicious cycle was often difficult through traditional means.

Findings from the recent cardiovascular outcome trials have demonstrated that SGLT2 inhibitors reduced hospitalization for HF by $27-35 \%$ in patients with T2DM with or without CVD and with or without a history of HF (5-7). SGLT2 inhibitors also reduced the composite of cardiovascular death or hospitalization for heart failure by $29-31 \%$ in patients with CKD with or without T2DM, CVD or a history of $\operatorname{HF}(25,26)$. In the DAPA-CKD trial, this cardiovascular benefit was consistent between diabetic (HR 0.70, 95\% CI 0.53-0.92) and non-diabetic (HR 0.79, 95\% CI $0.40-1.55)$ patients $\left(p_{\text {interaction }}=0.78\right)(27)$. The benefit of SGLT2 inhibitors in reducing the risk of HF was confirmed in the DAPAHF and EMPEROR-Reduced trials, which evaluated the effect of dapagliflozin and empagliflozin, respectively, in patients with $\mathrm{HF}$ and a reduced ejection fraction irrespective of T2DM diagnosis $(8,28)$. In the DAPA-HF trial, over a median of 18.2 months, the risk of a composite of worsening HF (hospitalization or an urgent visit requiring intravenous therapy for $\mathrm{HF}$ ) or cardiovascular death was reduced by $26 \%$ among patients receiving dapagliflozin when compared with the placebo group (HR 0.74, 95 CI 0.65$0.85, p<0.001$ ) (8). After a median follow-up of 16 months, empagliflozin reduced the risk of a composite of cardiovascular death or hospitalization for HF by $25 \%$ vs. placebo (HR 0.75 , 95\% CI $0.65-0.86, p<0.001$ ) (28). The lower rate of the composite of cardiovascular death or hospitalization for $\mathrm{HF}$ in the dapagliflozin vs. placebo group in the DECLARE-TIMI 58 trial (4.9 vs. $5.8 \%$; HR $0.83,95 \%$ CI $0.73-0.95, p=0.005$ ) was found to be due to the $27 \%$ reduction in relative risk of hospitalization for HF (HR 0.73, 95\% CI 0.61-0.88) (7). It seems 


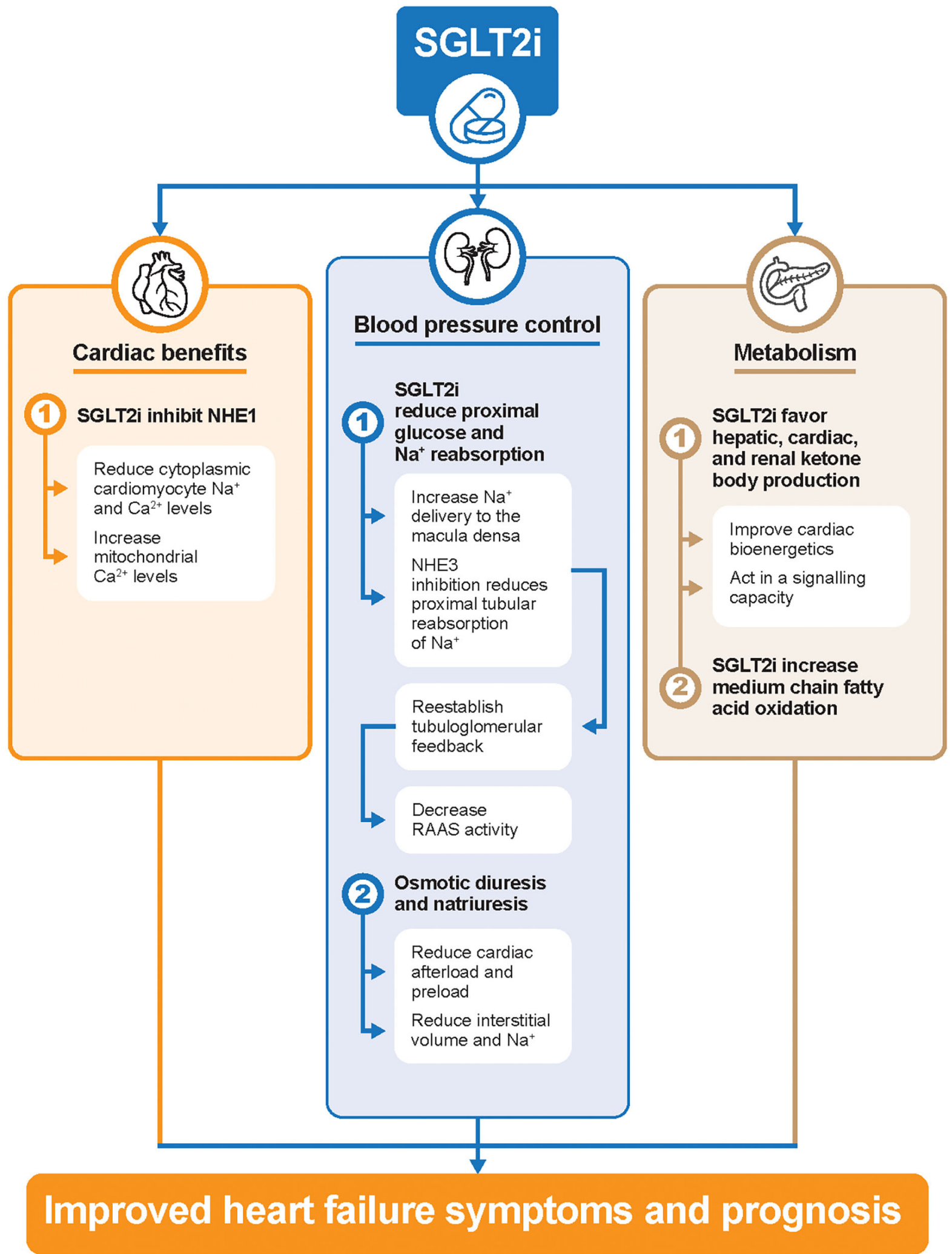

FIGURE 1 | Potential cardiorenal protection mechanisms of SGLT2 inhibitors in patients with HF. Ca2+, calcium; HF, heart failure; Na+, sodium; NHE, Na+/H+ exchanger; RAAS, renin-angiotensin-aldosterone system; SGLT2i, sodium-glucose cotransporter-2 inhibitors. 
that SGLT2 inhibitors have their greatest and most consistent effect on reducing the relative risk of hospitalization for HF. The beneficial effect of SGLT2 inhibitors in patients with HFpEF is currently under investigation (i.e., in the EMPOROR-Preserved, PRESERVED-HF and DELIVER trials).

Meanwhile, these cardiovascular outcomes trials have also demonstrated that SGLT2 inhibitors may be renoprotective, slowing the progression of renal disease in patients with or without T2DM (7, 25-27). Notably, dapagliflozin reduced the composite of worsening of renal function, end-stage renal disease, or renal or CVD death by $39 \%$ vs. placebo in patients the DAPA-CKD trial (HR 0.61, 95\% CI 0.51-0.72, $p<0.001$ ) (26). This finding was consistent between diabetic (HR 0.64, 95\% CI 0.52-0.79) and non-diabetic (HR 0.50, 95\% CI 0.35-0.72) patients $\left(p_{\text {interaction }}=0.24\right)(27)$. However, the incidence of the pre-specified renal composite outcome in the DAPA-HF trial, defined as a decline in estimated GFR, end-stage renal disease, or renal death, was comparable between the two treatment

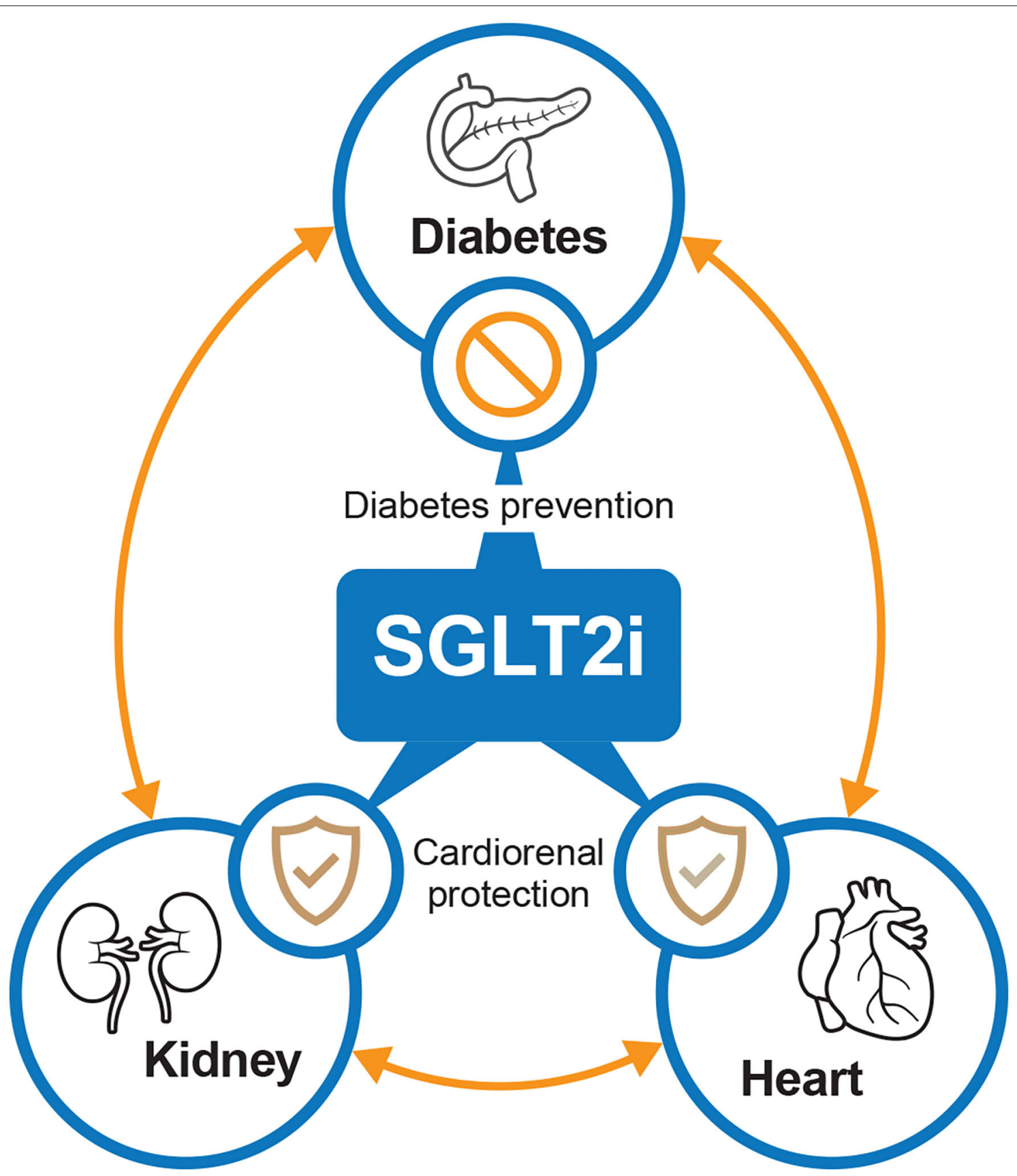

FIGURE 2 | The potential interplay between SGLT2 inhibitors, diabetes, kidney, and heart. SGLT2i, sodium-glucose cotransporter-2 inhibitors. 
groups (8). While promising, further evidence is required to confirm the renal benefit of SGLT2 inhibitors.

Overall, by breaking the vicious diabetes-kidney-heart cycle, the collective impact of SGLT2 inhibitors on heart and kidney function could explain why these agents may benefit all populations rather than only patients with T2DM.

\section{Mechanisms of Cardiorenal Protection by SGLT2 Inhibitors}

Despite extensive exploratory analyses, the exact mechanisms of the salutary effects of SGLT2 inhibitors remain unclear. The reduction in glycated hemoglobin due to SGLT2 inhibitors was approximately $0.5-1.0 \%$ (29), and it seems that glucose control itself more clearly translates into a reduction of microvascular rather than macrovascular complications (30). Instead, cardiorenal protection by SGLT2 inhibitors could be explained through the following mechanisms (Figure 1). First, SGLT2 inhibitors reduce glucose and sodium reabsorption in the proximal tubule, thus increasing sodium delivery to the macula densa (31). This, in turn, restores the tubuloglomerular feedback and increases RAAS activity $(31,32)$. Osmotic diuresis and natriuresis can also lead to a reduction of plasma volume (33). Second, SGLT2 inhibitors may directly inhibit the $\mathrm{Na}^{+} / \mathrm{H}^{+}$ exchanger (NHE) 1 isoform in the myocardium, which in turn reduces cytoplasmic sodium and calcium amounts and correspondingly increases mitochondrial calcium levels (34). Natriuresis promoted by SGLT2 inhibitors has also been accounted for by the downregulation of NHE3 activity in the proximal tubules (34). Overall, the inhibition of NHE may restore whole-body sodium homeostasis and reduce cardiac failure (34). Lastly, previous data showed that SGLT2 inhibitors shift myocardial fuel metabolism away from fat oxidation toward ketone bodies (35). However, other data have shown increased short-medium chain fatty acid oxidation is responsible for cardiac metabolic remodeling, and elevated plasma ketone bodies

\section{REFERENCES}

1. Savarese G, Lund LH. Global public health burden of heart failure. Card Fail Rev. (2017) 3:7-11. doi: 10.15420/cfr.2016:25:2

2. Velagaleti RS, Vasan RS. Heart failure in the twenty-first century: is it a coronary artery disease or hypertension problem? Cardiol Clin. (2007) 25:487-95. doi: 10.1016/j.ccl.2007. 08.010

3. Cheng YJ, Imperatore G, Geiss LS, Saydah SH, Albright AL, Ali MK, et al. Trends and disparities in cardiovascular mortality among U.S. adults with and without self-reported diabetes, 1988-2015. Diabetes Care. (2018) 41:2306-15. doi: 10.2337/dc18-0831

4. Scirica BM, Mosenzon O, Bhatt DL, Udell JA, Steg PG, McGuire DK, et al. Cardiovascular outcomes according to urinary albumin and kidney disease in patients with type 2 diabetes at high cardiovascular risk: observations from the SAVOR-TIMI 53 trial. JAMA Cardiol. (2018) 3:15563. doi: 10.1001/jamacardio.2017.4228

5. Zinman B, Wanner C, Lachin JM, Fitchett D, Bluhmki E, Hantel S, et al. Empagliflozin, cardiovascular outcomes, and mortality in type 2 diabetes. N Engl J Med. (2015) 373:2117-28. doi: 10.1056/NEJMoa150 4720 may play additional important signaling roles in improving cardiac performances (36-38).

In summary, SGLT2 inhibitors have provided rapid and significant improvements in cardio-renal outcomes (Figure 2). Specifically, demonstrable benefits in both the prevention and treatment of HF have been observed after SGLT2 inhibitor treatment. In addition, for patients with preserved renal function, SGLT2 inhibitors may represent an effective option to delay the progression of kidney disease. In the longterm, the retention of renal function can improve future cardiovascular-related outcomes by reducing the risk of both cardiovascular and all-cause death. Thus, SGLT2 inhibitors should be considered as a first-line option to decouple the diabetes-kidney-heart continuum. In other words, a new era of integrated management for HF, renal insufficiency, and T2DM is on the horizon, in which SGLT2 inhibitors will play an indispensable role.

\section{AUTHOR CONTRIBUTIONS}

YL contributed to the conceptualization of the article. All authors were involved in writing the original draft, all reviewed and edited the manuscript, and all read and approved the final manuscript.

\section{FUNDING}

The development of the commentary and editorial assistance was funded by AstraZeneca. The sponsor had no role in the conception, design, analysis, and interpretation of this manuscript or the decision to submit the report for publication.

\section{ACKNOWLEDGMENTS}

Editorial writing support, under the direction of the authors, was provided by Nucleus Global, Shanghai, China, funded by AstraZeneca in accordance with Good Publication Practice (GPP3) guidelines.

6. Perkovic V, Jardine MJ, Neal B, Bompoint S, Heerspink HJL, Charytan DM, et al. Canagliflozin and renal outcomes in type 2 diabetes and nephropathy. $N$ Engl J Med. (2019) 380:2295-306. doi: 10.1056/NEJMoa1811744

7. Wiviott SD, Raz I, Bonaca MP, Mosenzon O, Kato ET, Cahn A, et al. Dapagliflozin and cardiovascular outcomes in type 2 diabetes. $N$ Engl J Med. (2019) 380:347-57. doi: 10.1056/NEJMoa1812389

8. McMurray JJV, Solomon SD, Inzucchi SE, Kober L, Kosiborod MN, Martinez FA, et al. Dapagliflozin in patients with heart failure and reduced ejection fraction. N Engl J Med. (2019) 381:1995-2008. doi: 10.1056/NEJMoa1911303

9. Zelniker TA, Wiviott SD, Raz I, Im K, Goodrich EL, Bonaca MP, et al. SGLT2 inhibitors for primary and secondary prevention of cardiovascular and renal outcomes in type 2 diabetes: a systematic review and meta-analysis of cardiovascular outcome trials. Lancet. (2019) 393:31-9. doi: 10.1016/s0140-6736(18)32590-x

10. Dei Cas A, Khan SS, Butler J, Mentz RJ, Bonow RO, Avogaro A, et al. Impact of diabetes on epidemiology, treatment, and outcomes of patients with heart failure. JACC Heart Fail. (2015) 3:136-45. doi: 10.1016/j.jchf.2014.08.004

11. Marwick TH, Ritchie $\mathrm{R}$, Shaw JE, Kaye D. Implications of underlying mechanisms for the recognition and management of diabetic cardiomyopathy. J Am Coll Cardiol. (2018) 71:339-51. doi: 10.1016/j.jacc.2017.11.019 
12. Fotos NV, Giakoumidakis K, Kollia Z, Galanis P, Copanitsanou P, Pananoudaki E, et al. Health-related quality of life of patients with severe heart failure. A cross-sectional multicentre study. Scand J Caring Sci. (2013) 27:686-94. doi: 10.1111/j.1471-6712.2012.01078.x

13. Johansson I, Dahlström $U$, Edner $M$, Näsman $P$, Rydén L, Norhammar A. Prognostic implications of type 2 diabetes mellitus in ischemic and nonischemic heart failure. J Am Coll Cardiol. (2016) 68:1404-16. doi: 10.1016/j.jacc.2016.06.061

14. Pocock SJ, Ariti CA, McMurray JJV, Maggioni A, Køber L, Squire IB, et al. Predicting survival in heart failure: a risk score based on 39372 patients from 30 studies. Eur Heart J. (2012) 34:1404-13. doi: 10.1093/eurheartj/ehs337

15. Sharp TE III, Lefer DJ, Houser SR. Cardiometabolic heart failure and HFpEF: still chasing unicorns. JACC Basic Transl Sci. (2019) 4:4224. doi: $10.1016 /$ j.jacbts.2019.05.003

16. Fineberg D, Jandeleit-Dahm KA, Cooper ME. Diabetic nephropathy: diagnosis and treatment. Nat Rev Endocrinol. (2013) 9:71323. doi: $10.1038 /$ nrendo. 2013.184

17. Jia G, Whaley-Connell A, Sowers JR. Diabetic cardiomyopathy: a hyperglycaemia- and insulin-resistance-induced heart disease. Diabetologia. (2018) 61:21-8. doi: 10.1007/s00125-017-4390-4

18. Cherney DZ, Perkins BA, Soleymanlou N, Maione M, Lai V, Lee A, et al. Renal hemodynamic effect of sodium-glucose cotransporter 2 inhibition in patients with type 1 diabetes mellitus. Circulation. (2014) 129:58797. doi: 10.1161/circulationaha.113.005081

19. Takenaka $\mathrm{T}$, Inoue $\mathrm{T}$, Watanabe $\mathrm{Y}$. How the kidney hyperfiltrates in diabetes: from molecules to hemodynamics. World J Diabetes. (2015) 6:57682. doi: 10.4239/wjd.v6.i4.576

20. De Cosmo S, Viazzi F, Pacilli A, Giorda C, Ceriello A, Gentile S, et al. Predictors of chronic kidney disease in type 2 diabetes: a longitudinal study from the AMD annals initiative. Medicine. (2016) 95:e4007. doi: 10.1097/md.0000000000004007

21. Fu H, Liu S, Bastacky SI, Wang X, Tian XJ, Zhou D. Diabetic kidney diseases revisited: a new perspective for a new era. Mol Metab. (2019) 30:25063. doi: 10.1016/j.molmet.2019.10.005

22. Guo Y, Cui L, Ye P, Li J, Wu S, Luo Y. Change of kidney function is associated with all-cause mortality and cardiovascular diseases: results from the Kailuan study. J Am Heart Assoc. (2018) 7:e010596. doi: 10.1161/jaha.118. 010596

23. Schefold JC, Filippatos G, Hasenfuss G, Anker SD, von Haehling S. Heart failure and kidney dysfunction: epidemiology, mechanisms and management. Nat Rev Nephrol. (2016) 12:610-23. doi: 10.1038/nrneph.2 016.113

24. Mullens W, Abrahams Z, Francis GS, Sokos G, Taylor DO, Starling RC, et al. Importance of venous congestion for worsening of renal function in advanced decompensated heart failure. J Am Coll Cardiol. (2009) 53:58996. doi: 10.1016/j.jacc.2008.05.068

25. Neal B, Perkovic V, Mahaffey KW, de Zeeuw D, Fulcher G, Erondu $\mathrm{N}$, et al. Canagliflozin and cardiovascular and renal events in type 2 diabetes. N Engl J Med. (2017) 377:644-57. doi: 10.1056/NEJMoa161 1925

26. Heerspink HJL, Stefánsson BV, Correa-Rotter R, Chertow GM, Greene T, Hou F-F, et al. Dapagliflozin in patients with chronic kidney disease. New Engl J Med. (2020) 383:1436-46. doi: 10.1056/NEJMoa2024816

27. Wheeler DC, Stefánsson BV, Jongs N, Chertow GM, Greene T, Hou FF, et al. Effects of dapagliflozin on major adverse kidney and cardiovascular events in patients with diabetic and non-diabetic chronic kidney disease: a prespecified analysis from the DAPA-CKD trial. Lancet Diabetes Endocrinol. (2021) 9:22-31. doi: 10.1016/s2213-8587(20)30369-7
28. Packer M, Anker SD, Butler J, Filippatos G, Pocock SJ, Carson P, et al. Cardiovascular and renal outcomes with empagliflozin in heart failure. $N$ Engl J Med. (2020) 383:1413-24. doi: 10.1056/NEJMoa2022190

29. Zelniker TA, Braunwald E. Cardiac and renal effects of sodium-glucose cotransporter 2 inhibitors in diabetes: JACC state-of-the-art review. J Am Coll Cardiol. (2018) 72:1845-55. doi: 10.1016/j.jacc.2018.06.040

30. Patel A, MacMahon S, Chalmers J, Neal B, Billot L, Woodward M, et al. Intensive blood glucose control and vascular outcomes in patients with type 2 diabetes. N Engl J Med. (2008) 358:2560-72. doi: 10.1056/NEJMoa0802987

31. Fioretto P, Zambon A, Rossato M, Busetto L, Vettor R. SGLT2 inhibitors and the diabetic kidney. Diabetes Care. (2016) 39(Suppl. 2):S165-71. doi: 10.2337/dcS15-3006

32. Schork A, Saynisch J, Vosseler A, Jaghutriz BA, Heyne N, Peter A, et al. Effect of SGLT2 inhibitors on body composition, fluid status and renin-angiotensin-aldosterone system in type 2 diabetes: a prospective study using bioimpedance spectroscopy. Cardiovasc Diabetol. (2019) 18:46. doi: 10.1186/s12933-019-0852-y

33. Bertero E, Prates Roma L, Ameri P, Maack C. Cardiac effects of SGLT2 inhibitors: the sodium hypothesis. Cardiovasc Res. (2018) 114:128. doi: $10.1093 / \mathrm{cvr} / \mathrm{cvx} 149$

34. Verma S, McMurray JJV. SGLT2 inhibitors and mechanisms of cardiovascular benefit: a state-of-the-art review. Diabetologia. (2018) 61:2108-17. doi: 10.1007/s00125-018-4670-7

35. Ferrannini E, Mark M, Mayoux E, CV. Protection in the EMPA-REG OUTCOME trial: a "thrifty substrate" hypothesis. Diabetes Care. (2016) 39:1108-14. doi: 10.2337/dc16-0330

36. Oh CM, Cho S, Jang JY, Kim H, Chun S, Choi M, et al. Cardioprotective potential of an SGLT2 inhibitor against doxorubicin-induced heart failure. Korean Circ J. (2019) 49:1183-95. doi: 10.4070/kcj.2019.0180

37. Schugar RC, Moll AR, André d'Avignon D, Weinheimer CJ, Kovacs A, Crawford PA. Cardiomyocyte-specific deficiency of ketone body metabolism promotes accelerated pathological remodeling. Mol Metab. (2014) 3:75469. doi: 10.1016/j.molmet.2014.07.010

38. Puchalska P, Crawford PA. Multi-dimensional roles of ketone bodies in fuel metabolism, signaling, and therapeutics. Cell Metab. (2017) 25:26284. doi: 10.1016/j.cmet.2016.12.022

Conflict of Interest: This study received funding from AstraZeneca. The funder had the following involvement with the study: funding development of the commentary and editorial assistance.

The authors declare that the research was conducted in the absence of any commercial or financial relationships that could be construed as a potential conflict of interest.

Publisher's Note: All claims expressed in this article are solely those of the authors and do not necessarily represent those of their affiliated organizations, or those of the publisher, the editors and the reviewers. Any product that may be evaluated in this article, or claim that may be made by its manufacturer, is not guaranteed or endorsed by the publisher.

Copyright (C) 2021 Bao, Gao, Xie and Li. This is an open-access article distributed under the terms of the Creative Commons Attribution License (CC BY). The use, distribution or reproduction in other forums is permitted, provided the original author(s) and the copyright owner(s) are credited and that the original publication in this journal is cited, in accordance with accepted academic practice. No use, distribution or reproduction is permitted which does not comply with these terms. 